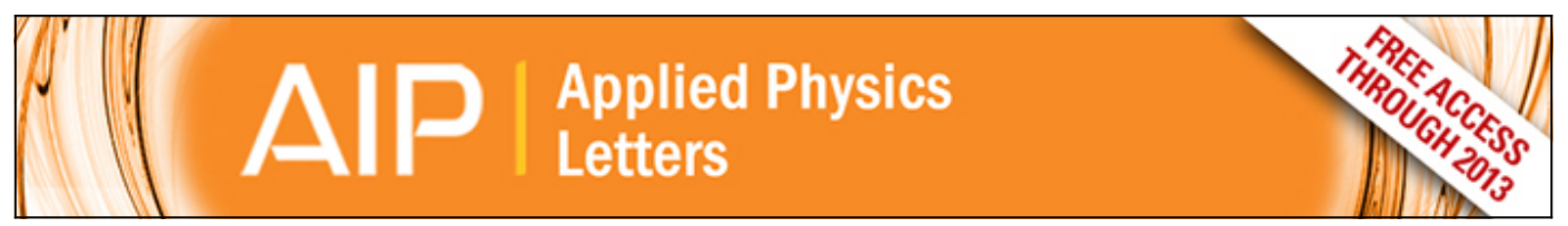

Correlation between thermal annealing temperature and Joule-heating based insulatormetal transition in VO2 nanobeams

Servin Rathi, Jin-Hyung Park, In-yeal Lee, Min Jin Kim, Jeong Min Baik, and Gil-Ho Kim

Citation: Applied Physics Letters 103, 203114 (2013); doi: 10.1063/1.4831995

View online: http://dx.doi.org/10.1063/1.4831995

View Table of Contents: http://scitation.aip.org/content/aip/journal/apl/103/20?ver=pdfcov

Published by the AIP Publishing

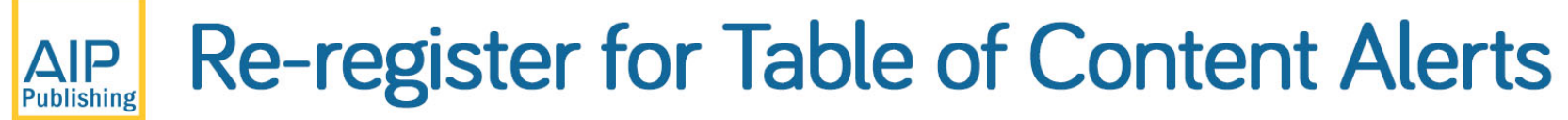




\title{
Correlation between thermal annealing temperature and Joule-heating based insulator-metal transition in $\mathrm{VO}_{2}$ nanobeams
}

\author{
Servin Rathi, ${ }^{1}$ Jin-Hyung Park, ${ }^{1}$ In-yeal Lee, ${ }^{1}$ Min Jin Kim, ${ }^{2}$ Jeong Min Baik, ${ }^{2}$ \\ and Gil-Ho Kim ${ }^{1, a)}$ \\ ${ }^{1}$ School of Electronic and Electrical Engineering and Sungkyunkwan Advanced Institute \\ of Nanotechnology (SAINT), Sungkyunkwan University, Suwon 440-746, South Korea \\ ${ }^{2}$ School of Mechanical and Advanced Materials Engineering, Ulsan National Institute of Science \\ and Technology (UNIST), Ulsan 689-805, South Korea
}

(Received 5 August 2013; accepted 3 November 2013; published online 15 November 2013)

\begin{abstract}
Rapid thermal annealing of $\mathrm{VO}_{2}$ nanobeams in an ambient argon environment has been carried out at various temperatures after device fabrication. Our analysis revealed that increasing the annealing temperature from $200^{\circ} \mathrm{C}$ to $400{ }^{\circ} \mathrm{C}$ results in the reduction of both ohmic and nanobeam resistances with an appreciable decrease in joule-heating based transition voltage and transition temperature, while samples annealed at $500^{\circ} \mathrm{C}$ exhibited a conducting rutile-phase like characteristics at room temperature. In addition, these variation trends were explored using a physical model and the results were found to be in agreement with the observed results, thus verifying the model. (C) 2013 AIP Publishing LLC. [http://dx.doi.org/10.1063/1.4831995]
\end{abstract}

Of all correlated transition metal oxides, the transition temperature $\left(T_{C} \cong 67{ }^{\circ} \mathrm{C}\right)$ of vanadium dioxide $\left(\mathrm{VO}_{2}\right)$ from an insulating to a metallic phase is closest to room temperature; therefore, its applications offer huge potential in devices such as switches and sensors. ${ }^{1,2}$ Below $T_{C}, \mathrm{VO}_{2}$ is a semiconductor (an energy band-gap $\left(E_{g}\right)$ of $0.6 \mathrm{eV}$ ) with a monoclinic crystal lattice, and above $T_{C}$, it behaves like a metal with a tetragonal (rutile) lattice. ${ }^{3,4}$ In sensing and switching applications, an accurate determination and control of transition point is at the heart of the device operations. ${ }^{5-9}$ Both thermal and joule heating $(\mathrm{JH})$ offer a simple approach to induce transition in the highly resistive $\mathrm{VO}_{2}$ nanobeams (NBs) as the requirement of $T_{C}$ can be readily met by either simply heating or applying a suitable voltage/current source. Besides domain boundaries shifting and interface stress with an underlying substrate, oxidation risks and the subsequent formation of various Magnéli phases $\left(\mathrm{V}_{\mathrm{n}} \mathrm{O}_{2 \mathrm{n}-1}=\mathrm{V}_{2} \mathrm{O}_{3}+(\mathrm{n}-2) \mathrm{VO}_{2}\right)$ during synthesis and processing can result in the crucial $T_{C}$ and $V_{T}$ (the voltage at which transition occurs, usually marked by a $3-5$ orders of abrupt current-jump) shifting. ${ }^{10-14}$ These shifts can be detrimental to device lifetimes (number of sensing or memory operations) and fail-safe operations. Previous studies based on $\mathrm{VO}_{2}$ thin films ${ }^{10-12}$ and $\mathrm{NBs}^{13,14}$ have indicated that $\mathrm{VO}_{2}$ has a strong tendency to exist in multiple valence states (Magnéli phases) and is therefore very sensitive to synthesis conditions and the subsequent device fabrication conditions such as post-fabrication annealing. Therefore, the effects of variation in the stoichiometry of $\mathrm{VO}_{2} \mathrm{NBs}$ on the critical parameters like $T_{C}$ and $V_{T}$ due to post-fabrication annealing temperature are the focus of this study. Moreover, different annealing temperatures have been reported for $\mathrm{VO}_{2} \mathrm{NBs}^{9,15}$ in the literature and the absence of any correlation study provides further motivation for this work.

The $\mathrm{VO}_{2} \mathrm{NBs}$ were grown by physical vapour deposition (PVD) technique, ${ }^{16}$ and the devices were fabricated by

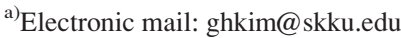

typical photolithography and lift-off techniques. Fig. 1 shows the scanning electron microscope (SEM) images of one such $\mathrm{VO}_{2} \mathrm{NB}$ device. In order to analyze the effects of annealing on $\mathrm{VO}_{2} \mathrm{NBs}$, rapid thermal annealing (RTA) was carried out in an ambient argon environment at a flow rate of $1000 \mathrm{sccm}$ and a pressure of 3.62 Torr at $200^{\circ} \mathrm{C}, 300^{\circ} \mathrm{C}, 400^{\circ} \mathrm{C}$, and $500{ }^{\circ} \mathrm{C}$ for $1 \mathrm{~min}$. As shown in Fig. 2(a), the variation in the total resistance, including individual contributions from both ohmic contacts and NBs, were measured at room temperature for different annealing conditions. The division of total resistance was carried out using the four-probe technique. It can be seen from the figure that increasing the annealing temperature results in the reduction of both $\mathrm{NB}$ and ohmic contact resistances. The measurement was repeated on at least 6 devices for each category and the error bars in the figure indicate the variation in the total resistance for multiple devices. These variations are likely to be caused by the fluctuations in the density of random defects, interstitials, and vacancies formed in $\mathrm{VO}_{2} \mathrm{NBs}$ during the PVD process. The ohmic contribution drops from $25 \%$ in the pre-annealed device to less than $0.5 \%$ in both D200 (device annealed at $200^{\circ} \mathrm{C}$ ) and D300 and becomes negligible for D400 and D500. The average resistivity of $\mathrm{VO}_{2} \mathrm{NBs}$ varies from $1.6 \Omega$ $\mathrm{cm}, 4.5 \times 10^{-1} \Omega \mathrm{cm}, 2.2 \times 10^{-1} \Omega \mathrm{cm}, 3.8 \times 10^{-2} \Omega \mathrm{cm}$, $9.0 \times 10^{-4} \Omega \mathrm{cm}$ for the pre-annealed, D200, D300, D400, and D500, respectively. While resistivity of all the devices, except D500, falls within the reported range, ${ }^{2,3,5,7}$ the ultra-low resistivity value of D500, most likely indicating a metallic behaviour, ${ }^{3}$ would be discussed later. As far as the quality of ohmic contact is concerned, the straight line I-V curves in Fig. 2(b) show good ohmic features not only for D200 but for the pre-annealed device as well. Therefore, the observed reduction in the ohmic resistance with high temperature annealing conditions would have originated either from the enhanced metal-NB surface contact area or due to lowering of $\mathrm{VO}_{2}$ work-function in the high temperature reducing environment ${ }^{17}$ which can affect NB and metal workfunction $^{18}$ based barrier height and hence the ohmic resistance. 

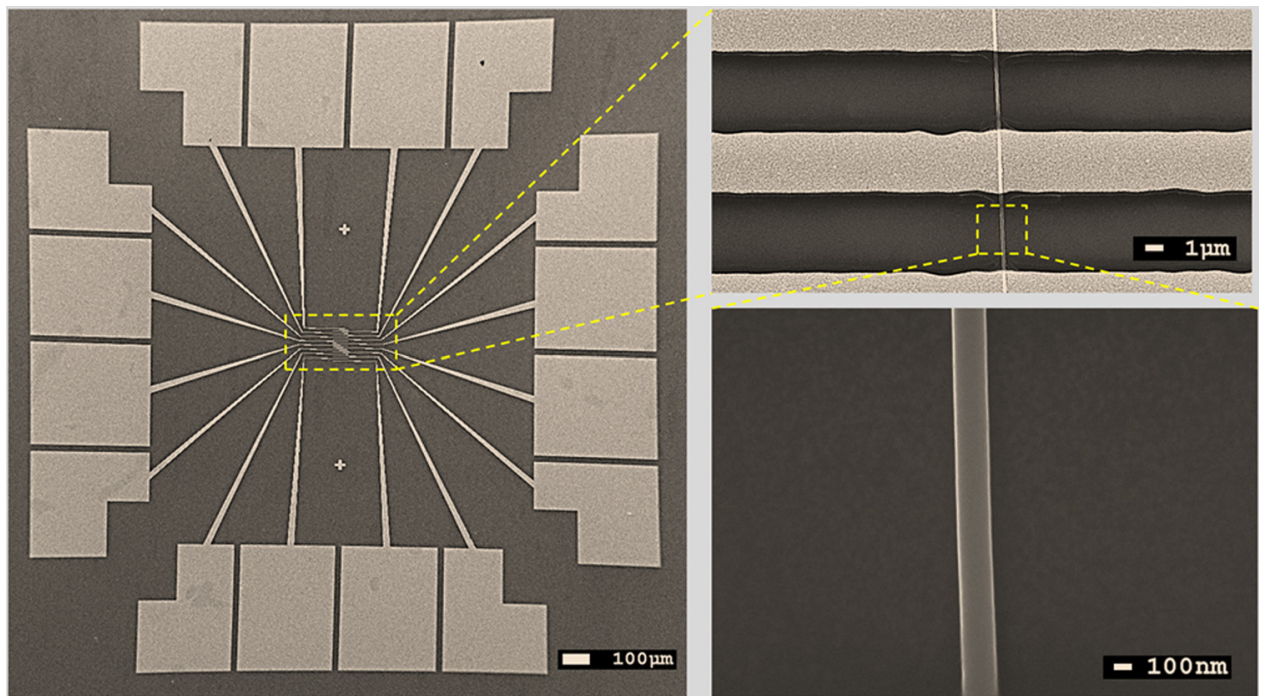

FIG. 1. SEM image of a patterned metal electrodes chip with a $\mathrm{VO}_{2} \mathrm{NB}$ crossing through the pattern.

In order to study the impact of annealing conditions on the $\mathrm{JH}$ based transition voltage, $V_{T}$, typical voltage hysteresis curves are plotted in Fig. 3. The measurements were carried
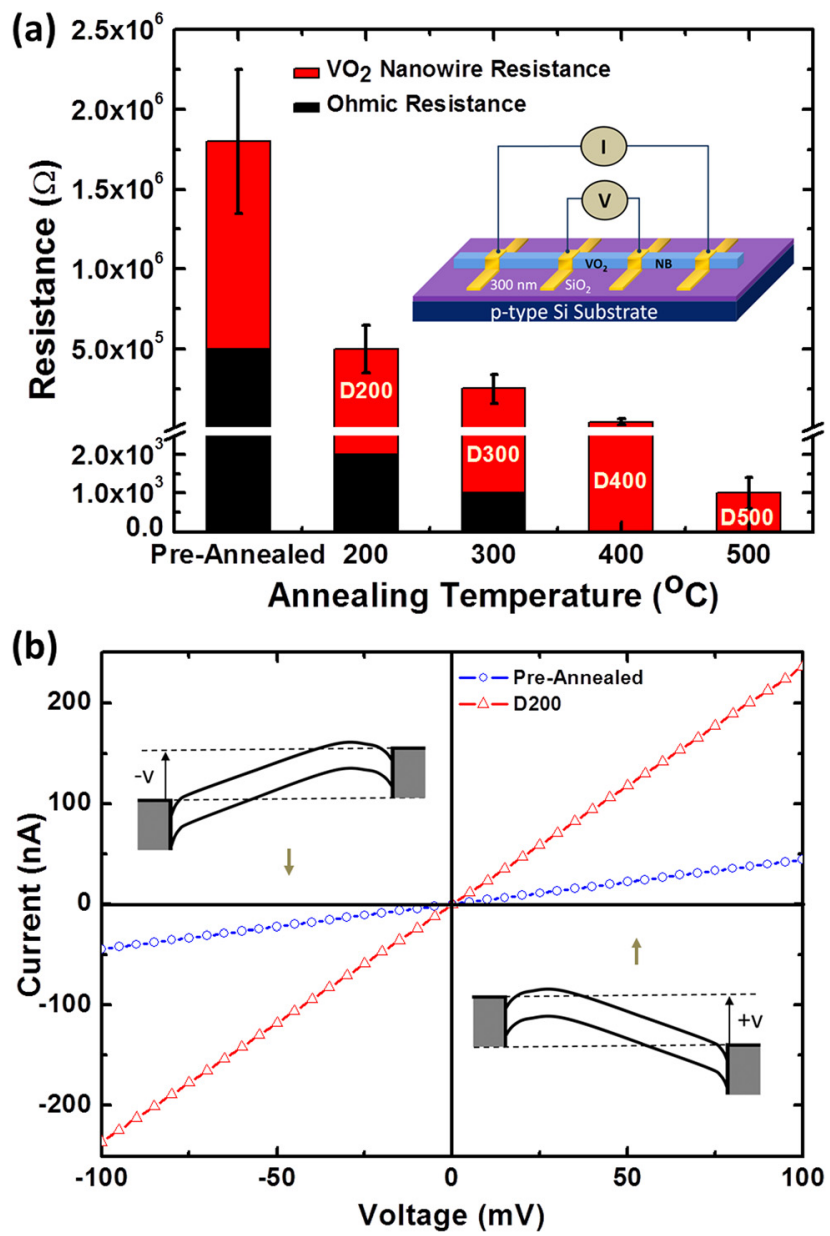

FIG. 2. Four-probe and two-probe measurement of $\mathrm{VO}_{2} \mathrm{NBs}$. (a) Resistance variation measured at room temperature following $\mathrm{RTA}$ at $200^{\circ} \mathrm{C}, 300^{\circ} \mathrm{C}$, $400{ }^{\circ} \mathrm{C}$, and $500^{\circ} \mathrm{C}$ for $1 \mathrm{~min}$ in an ambient argon environment. The error bars show resistance variation for multiple devices and the schematic in the inset illustrates the four probe measurement setup. (b) I-V curves of pre-annealed and D200 at room temperature. The schematics in the inset show band diagrams for two-terminal device, considering 5.33 eV (Ref. 17) and 5.1 eV (Ref. 18) as workfunction of $\mathrm{VO}_{2} \mathrm{NB}$ and metal-electrodes, respectively. out at an ambient temperature of $47^{\circ} \mathrm{C}$ using a Keithley 4200 SCS measurement system with an upper current compliance of $1.0 \mathrm{~mA}$ which was set to avoid thermal burn-out of the NBs due to excessive current flow after the transition. It can be seen from the figure that increasing the annealing temperature resulted in the reduction of $V_{I M T}$, voltage at which NBs convert from insulator to metal, from $4.8 \mathrm{~V}$ to $2.1 \mathrm{~V}$ and $0.8 \mathrm{~V}$ for D200, D300, and D400, respectively. A similar reduction can also be observed for $V_{M I T}$, voltage at which the $\mathrm{VO}_{2} \mathrm{NBs}$ convert from metal to insulator. Although annealing affects $V_{T}$ (both $V_{I M T}$ and $V_{M I T}$ ) of the devices, all the devices maintained a proportionate voltage-triggered hysteresis window, a typical characteristic feature of $\mathrm{VO}_{2} \mathrm{NBs}$ and thin films devices. Before elaborating further on these variations, the hysteretic temperature-triggered transition of an annealed device is plotted in Fig. 4. The measurement was carried out with a minimum accuracy of $\pm 2{ }^{\circ} \mathrm{C}$ and the $T_{C}$ was calculated as the mean of $T_{I M T}$ and $T_{M I T}$, which were extracted from the minima of $\ln R$ vs $T$ for heating and cooling branches, respectively. The inset in Fig. 4 shows $\mathrm{VO}_{2}$ energy band diagrams based on

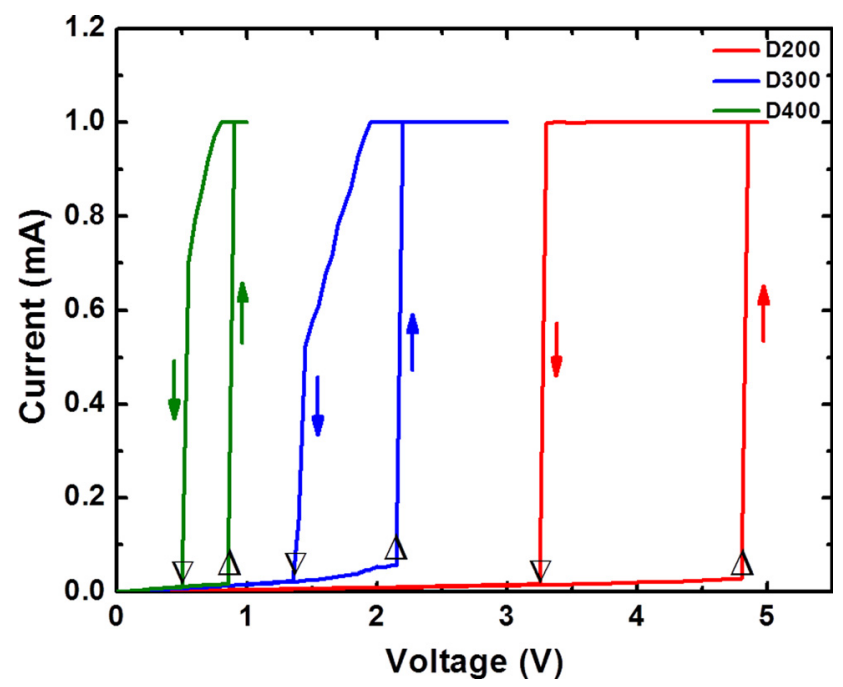

FIG. 3. JH based transition voltages, $V_{I M T}$ and $V_{M I T}$, variations at an ambient temperature of $47^{\circ} \mathrm{C}$ of D200, D300, and D400 (symbols $\triangle$ and $\nabla$ indicate $V_{I M T}$ and $V_{M I T}$, respectively). 


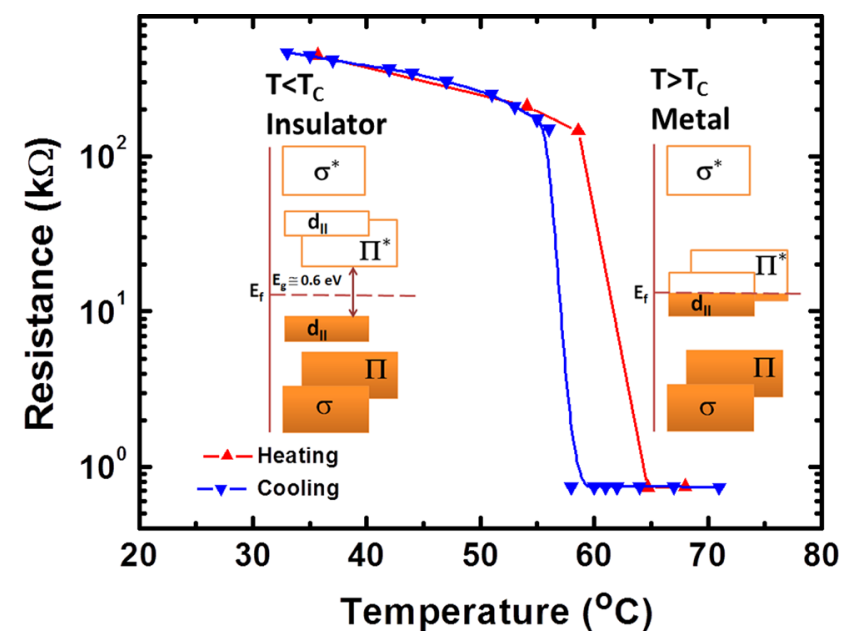

FIG. 4. Resistance versus temperature curve of an annealed device; the insets show $\mathrm{VO}_{2}$ energy band diagrams for both insulating and metallic states.

the molecular orbital and crystal field theory employed by Goodenough, ${ }^{19}$ where $d_{l l}$ band splits as $\mathrm{VO}_{2}$ undergoes transition from a metal to an insulating state. ${ }^{1,19,20}$ The hysteresis observed in both $\mathrm{JH}$ (Fig. 3) and thermal induced transition (Fig. 4) is a characteristic feature of the first order phase transition, which in general, requires some amount of overheating (undercooling) above (below) the equilibrium transition temperature, where the energy curves of the two phases (monoclinic and tetragonal) intersect with a discontinuity in the first derivative. 6,21 However, at thermodynamic equilibrium, the system occupies one of the two (meta) stable states depending on the transformation history, i.e., whether the temperature has been increasing or decreasing. ${ }^{21}$

As $\mathrm{JH}$ involves heat dissipation due to current flow across a resistive material, $\mathrm{JH}$ based transition as observed in Fig. 3 indicates that the transition occurred when the local temperature in the NB reaches the critical value, $T_{C}$. Thus, $\mathrm{JH}$ based transition voltage depends on both resistance and $T_{C}$ of the NB and therefore any change in transition voltages (both $V_{I M T}$ and $V_{M I T}$ ) could be either due to the variation in NB's resistance or $T_{C}$ or both. A physical model ${ }^{5}$ for $\mathrm{VO}_{2}$ $\mathrm{NBs}$ is employed to ascertain the main factors behind this observed $V_{I M T}$ and $V_{M I T}$ variations. According to the model, power dissipated as joule heating in a NB is counterbalanced by conductive losses through the ambient environment, metal electrodes, and the substrate and can be expressed by the following equation: ${ }^{5}$

$$
\frac{d Q}{d t}=\frac{V_{c}^{2}}{R_{c}}-k\left(T-T_{o}\right),
$$

where $k$ is a collection of thermal parameters, including the thermal coefficient and heat capacity of the NB and the underlying substrate surface, $V_{c}$ and $R_{c}$ are the voltage and resistance across the NB, respectively, and $T$ and $T_{o}$ are the temperature of NB and ambient environment, respectively. For a NB with its temperature approaching $T_{C}$, Eq. (1) can be solved for the steady-state condition $\left(\frac{d Q}{d t}=0\right)$, and it can be further simplified for negligible ohmic contact resistance by the following expression:

$$
V_{I M T}=\left(k\left(T_{I M T}-T_{o}\right) R_{A}\right)^{1 / 2}
$$

where $R_{A}$ is the NB resistance before transition. Eq. (2) gives the factors affecting $V_{I M T}$, including the resistance (dimensions and resistivity) of the $\mathrm{NBs}, T_{C}$, and the ambient temperature. A similar analogous expression for the $V_{M I T}$, at which reverse transition occurs when voltage is swept from high to low, can be deduced

$$
V_{M I T}=\left(k^{\prime}\left(T_{M I T}-\mathrm{T}_{\mathrm{o}}\right) R_{B}\right)^{1 / 2},
$$

where $k^{\prime}$ is the collection of thermal coefficients in the metallic phase at high temperature, and $R_{B}$ is the NB resistance in the metallic (rutile) phase. If we assume a constant transition temperature $\left(T_{C}\right)$ independent of annealing conditions, then according to Eq. (2), variation in $V_{I M T}$ should follow the NB resistance. This is acceptable in the case of $V_{I M T}$, where reduction in NB resistance can directly lead to higher joule heating thus lower $V_{I M T}$. But the constant resistance of $1 \mathrm{k} \Omega$ approximately ${ }^{16}\left(R_{B}\right)$ in the rutile phase and the declining trend in $V_{M I T}$ values does not support the constant $T_{C}$ assumption.

To resolve this, the difference of the square of Eqs. (2) and (3) was considered, neglecting the terms in the metallic phase (owing to the very small value of resistance compared to the insulating phase), and replacing $T_{I M T}$ with the mean value $^{5}\left(T_{C}\right)$, it gives

$$
\begin{aligned}
V_{I M T}^{2}-V_{M I T}^{2}= & \Delta V_{M}^{2}=k\left(T_{I M T}-T_{o}\right) R_{A}-k^{\prime} \\
& \times\left(T_{M I T}-T_{o}\right) R_{B} \cong k\left(T_{C}-T_{o}\right)\left(R_{A}\right) .
\end{aligned}
$$

The ratios of $\Delta V_{M}^{2}$ and $R_{A}$ were calculated as 4.29, 2.81 and 23.14, 3.55 for D200/D300 and D200/D400, respectively. This considerable mismatch obtained between the ratios of $\Delta V_{M}^{2}$ and $R_{A}$ clearly indicates the variation in $T_{C}$ with annealing temperature. And the altered $T_{C}$ of the annealed device can be deduced using the following equation:

$$
\frac{\left(\Delta V_{M}^{2}\right)_{D 200}}{\left(\Delta V_{M}^{2}\right)_{D 300}}=\frac{\left(k\left(T_{C}-T_{o}\right) R_{A}\right)_{D 200}}{\left(k\left(T_{C}-T_{o}\right) R_{A}\right)_{D 300}} .
$$

Using a similar equation for D400 as well and assuming $T_{C}=67^{\circ} \mathrm{C}$ for D200 device, $T_{C}$ for D300 and D400 was calculated to be $60.1^{\circ} \mathrm{C}$ and $50.1^{\circ} \mathrm{C}$, respectively. The measured and modeled values of $T_{C}$ are plotted in Fig. 5(a). The error bars indicate the variation in $T_{C}$ for multiple devices of each category. Although the $T_{C}$ variation between D200 and D300 was small, a decent depression in the case of D400 agrees with the trend predicted by the physical model. Similar $T_{C}$ depression in $\mathrm{VO}_{2}$ crystals has also been reported previously, ${ }^{12,13,22-25}$ where it has been attributed to oxygen deficiency-related defects such as oxygen vacancies, resulting in non-stoichiometric $\mathrm{VO}_{2}$. These defects either act like electron dopants that generate free electrons or as lattice deformation sites that generates internal strain and may serve as the nucleation sites with lower free energy for first order phase transition ${ }^{26}$ in non-stoichiometric $\mathrm{VO}_{2}$ thus influencing the transition point. ${ }^{13}$ These propositions can be readily applied in this study as well, where annealing at high 

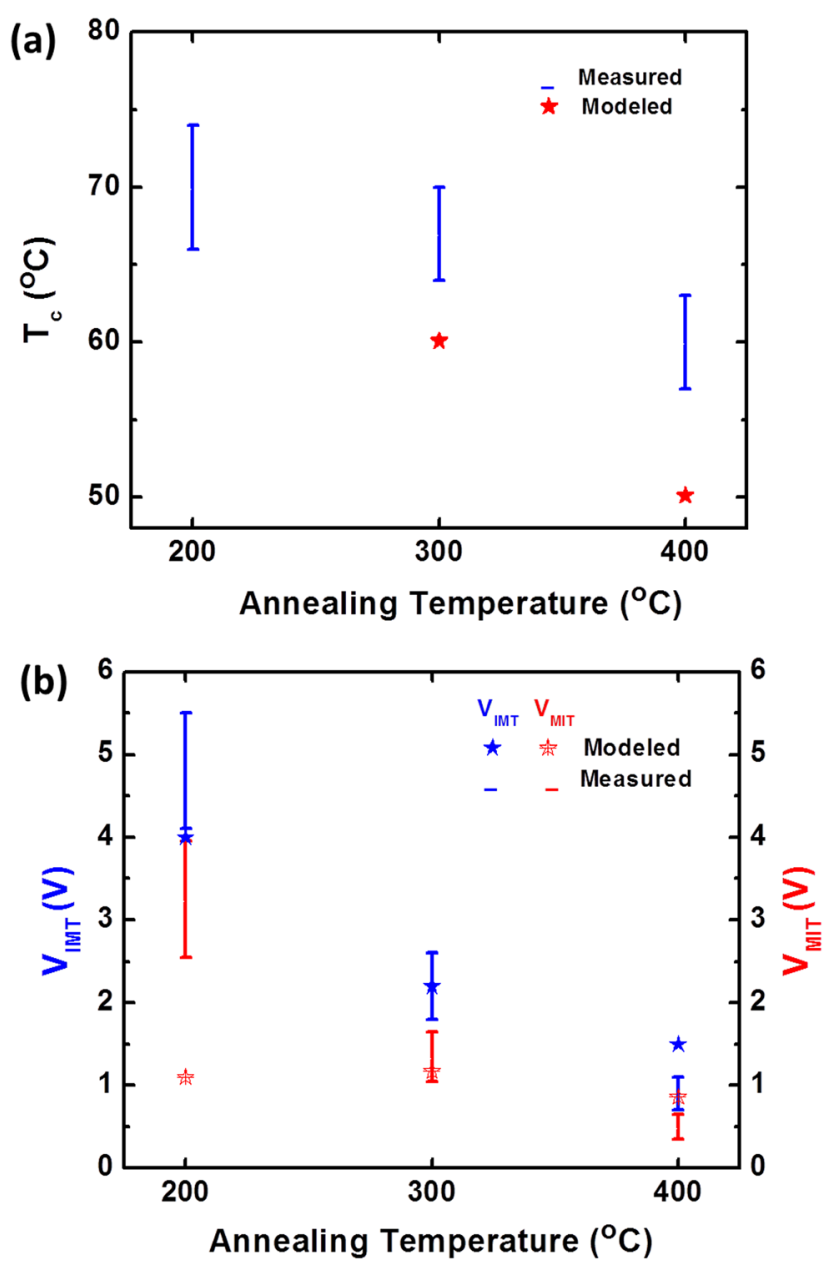

FIG. 5. Variations observed in the transition points. (a) Thermal transition $\left(T_{C}\right)$, (b) $\mathrm{JH}$ induced transition voltages $\left(V_{I M T}\right.$ and $\left.V_{M I T}\right)$ versus annealing temperature, with error bars indicating the variation obtained from multiple devices. The modeled values are also indicated by star symbols.

temperature can easily induce oxygen deficiency-related lattice defects and vacancies. This results in the reduction of NB resistance owing to a higher concentration of free electrons which in turn lowers the stabilization energy of the distorted monoclinic lattice thus resulting in $T_{C}$ reduction. Fig. 5(b) shows the observed $V_{I M T}$ and $V_{M I T}$ for different annealing temperature, with error bars showing variations for multiple devices annealed at the same temperature. Additionally, the observed $V_{I M T}$ and $V_{M I T}$ were also calculated using Eqs. (2) and (3), and the calculated values ${ }^{16}$ were found to be in agreement with the experimental values, thus proving the robustness of our simple model. At the end, the D500 device, which showed the rutile state behaviour on annealing (Fig. 2 ), can be similarly explained by an excess of oxygen deficiency-related defects (acting like electron dopants and/or lattice deformers), simultaneously resulting in higher conductivity and reduction in $T_{C}$, thus stabilizing the oxygen deficient non-stoichiometric rutile phases of $\mathrm{VO}_{2} \mathrm{NBs}$ even at room temperature. ${ }^{13,17}$

In conclusion, we studied $\mathrm{VO}_{2} \mathrm{NBs}$ for the detailed analysis of $V_{I M T}, V_{M I T}$, and $T_{C}$ at different annealing temperatures by both $\mathrm{JH}$ and thermal induced hysteresis curves.
With increasing the annealing temperature from $200{ }^{\circ} \mathrm{C}$ to $400{ }^{\circ} \mathrm{C}$, both ohmic and NBs resistance decreases, and at $500{ }^{\circ} \mathrm{C}$, NBs were found to be in metallic (rutile) phase. The observed variations in $V_{I M T}$ and $V_{M I T}$ were attributed to both variations in the resistivity and $T_{C}$. The reduction in $T_{C}$ was also predicted from a simple physical model. This study would be relevant in optimizing the annealing conditions for good ohmic contacts while considering the variation in $\mathrm{VO}_{2}$ NBs and consequently the characteristic $V_{T}$ and $T_{C}$, which are critical parameters for any $\mathrm{VO}_{2}$-based sensors, switches, or memory nanodevices.

This research was supported by basic science research program through the national research foundation of Korea funded by the ministry of education, science, and technology (2012R1A1A2042230).

${ }^{1}$ Z. Yang, C. Ko, and S. Ramanathan, Annu. Rev. Mater. Res. 41, 337 (2011).

${ }^{2}$ E. Strelcov, Y. Lilach, and A. Kolmakov, Nano Lett. 9, 2322 (2009).

${ }^{3}$ J. Wei, Z. Wang, W. Chen, and D. H. Cobden, Nat. Nanotechnol. 4, 420 (2009).

${ }^{4}$ T. L. Cocker, L. V. Titova, S. Fourmaux, G. Holloway, H.-C. Bandulet, D. Brassard, J.-C. Kieffer, M. A. E. Khakani, and F. A. Hegmann, Phys. Rev. B $\mathbf{8 5}, 155120$ (2012).

${ }^{5}$ J. M. Baik, M. H. Kim, C. Larson, A. M. Wodtke, and M. Moskovits, J. Phys. Chem. C. 112, 13328 (2008).

${ }^{6}$ M. Nakano, K. Shibuya, D. Okuyama, T. Hatano, S. Ono, M. Kawasaki, Y. Iwasa, and Y. Tokura, Nature 487, 459 (2012).

${ }^{7}$ J. W. Byon, M. B. Kim, M. H. Kim, S. Y. Kim, S. H. Lee, B. C. Lee, and J. M. Baik, J. Phys. Chem. C. 116, 226 (2012).

${ }^{8}$ J. Kim, C. Ko, A. Frenzel, S. Ramanathan, and J. E. Hoffman, Appl. Phys. Lett. 96, 213106 (2010).

${ }^{9}$ J. M. Baik, M. H. Kim, C. Larson, C. T. Yavuz, G. D. Stucky, A. M. Wodtke, and M. Moskovits, Nano Lett. 9, 3980 (2009).

${ }^{10}$ Z. Yang, C. Ko, and S. Ramanathan, J. Appl. Phys. 108, 073708 (2010).

${ }^{11}$ D. Ruzmetov, S. D. Senanayake, and S. Ramanathan, Phys. Rev. B 75, 195102 (2007).

${ }^{12}$ D. Ruzmetov, S. D. Senanayake, V. Narayanamurti, and S. Ramanathan, Phys. Rev. B 77, 195442 (2008).

${ }^{13}$ S. Zhang, I. S. Kim, and L. J. Lauhon, Nano Lett. 11, 1443 (2011).

${ }^{14}$ J. Y. Chou, J. L. Lensch-Falk, E. R. Hemesath, and L. J. Lauhon, J. Appl. Phys. 105, 034310 (2009).

${ }^{15}$ K. Liu, D. Fu, J. Cao, J. Suh, K. Wang, C. Cheng, D. Ogletree, H. Guo, S. Sengupta, A. Khan, C. W. Yeung, S. Salahuddin, M. M. Deshmukh, and J. Wu, Nano Lett. 12, 6272 (2012).

${ }^{16}$ See supplementary material at http://dx.doi.org/10.1063/1.4831995 for a detail method of $\mathrm{VO}_{2}$ nanobeams growth, device fabrication, Raman spectra analysis, and calculation of joule heating based transition points, $V_{I M T}$, $V_{M I T}$.

${ }^{17}$ W. K. Hong, J. B. park, J. Yoon, B. J. Kim, J. I. Sohn, Y. B. Lee, T. S. Bae, S. J. Chang, Y. S. Huh, B. Son, E. A. Stach, T. Lee, and M. E. Welland, Nano Lett. 13, 1822 (2013).

${ }^{18}$ H. B. Michaelson, J. Appl. Phys. 48, 4729 (1977).

${ }^{19}$ J. B. Goodenough, J. Solid State Chem. 3, 490 (1971).

${ }^{20}$ M. S. Grinolds, V. A. Lobastov, J. Weissenrieder, and A. H. Zewail, Proc. Natl. Acad. Sci. U.S.A. 103, 18427 (2006).

${ }^{21}$ E. U. Donev, R. Lopez, L. C. Feldman, and R. F. Haglund, Jr., Nano Lett. 9, 702 (2009).

${ }^{22}$ N. Kimizuka, M. Saeki, and M. Nakahira, Mater. Res. Bull. 5, 403 (1970).

${ }^{23}$ C. C. Y. Kwan, C. H. Griffith, and H. K. Eastwood, Appl. Phys. Lett. 20, 93 (1972).

${ }^{24}$ C. H. Griffith and H. K. Eastwood, J. Appl. Phys. 45, 2201 (1974).

${ }^{25}$ J. M. Booth and P. S. Casey, Phys. Rev. Lett. 103, 086402 (2009).

${ }^{26}$ K. Appavoo, D. Y. Lei, Y. Sonnefraud, B. Wang, S. T. Pantelides, S. A. Maier, and R. F. Haglund, Jr., Nano Lett. 12, 780 (2012). 